\title{
A simulation analysis of the microstructure of an order driven financial market with multiple securities and portfolio choices
}

\author{
ANDREA CONSIGLIO*, VALERIO LACAGNINA and ANNALISA RUSSINO \\ Dip. di Scienze Statistiche e Matematiche 'Silvio Vianelli', Università di Palermo, Italy
}

(Received 11 November 2003; in final form 18 November 2004)

\begin{abstract}
In this paper we propose an artificial market where multiple risky assets are exchanged. Agents are constrained by the availability of resources and trade to adjust their portfolio according to an exogenously given target portfolio. We model the trading mechanism as a continuous auction order-driven market. Agents are heterogeneous in terms of desired target portfolio allocations, but they are homogeneous in terms of trading strategies. We investigate the role played by the trading mechanism in affecting the dynamics of prices, trading volume and volatility. We show that the institutional setting of a double auction market is sufficient to generate a non-normal distribution of price changes and temporal patterns that resemble those observed in real markets. Moreover, we highlight the role played by the interaction between individual wealth constraints and the market frictions associated with a double auction system to determine the negative asymmetry of the stock returns distribution.
\end{abstract}

\section{Introduction}

We analyse the dynamics of the price formation process of a set of financial securities through the construction of an artificial continuous auction order-driven financial market with heterogeneous agents who trade to adjust their portfolio according to a target portfolio.

The dynamics of a financial market depends on the interactions between the rules defining the trading mechanism and the behavioural assumptions about the agents' population. Building an artificial market means to determine the trading rules that define the price formation process and to specify the trading strategies followed by the agents.

In recent years this methodology has gathered increasing attention in order to explain the statistical properties of empirical financial time series. Research in finance has produced a wide empirical evidence showing that deviations from the standard assumptions (stationary and IID normally distributed financial time series) are common and cannot be considered exceptional deviations from the standard model. A number of regularities (or stylized facts) have been observed, such as fat tails and excess

*Corresponding author. Email: consiglio@unipa.it kurtosis of the short-term log-returns distribution, persistence of volatility and of trading volume, and cross-correlations between volatility and trading volume (see Karpoff 1987, Gallant et al. 1992, Pagan 1996, Campbell et al. 1997, Engle and Patton 2001). This empirical evidence opened two fundamental research areas. First, a growing effort has been devoted to search for statistical models that can fit the data and, thus, provide a good description of the standard patterns observed in financial series. Second, it became essential to understand what generates the common characteristics of financial time series and, ultimately, to develop economic models capable of generating the complex behaviour that is observed. In particular, it became important to explain how the institutional setting in which agents interact, and the agents behavioural characteristics, affect market results.

Artificial market models are effective tools of analysis for identifying the set of elements needed to mimic the structure and the patterns of empirically observed financial time series. Using numerical simulation, they make it possible to analyse the impact of systems of interactions which would otherwise be analytically intractable. Namely, they allow to overcome the necessary simplifying assumptions of theoretical models (such as the need to restrict the analysis to cases with representative agents), 
and they open the possibility of studying the effect of the simultaneous interaction of alternative market designs and heterogeneous agents on price dynamics.

Previous agent-based simulators focused their attention on analysing the impact of groups of agents following different trading strategies (see LeBaron 1999, Routledge 1999, Brock et al. 2001, Chen et al. 2001, Chiarella and He 2001, LeBaron 2001, Farmer and Joshi 2002). Typically, the interaction between value traders (or fundamentalists) and trend chasers is studied in a single risky asset economy characterized by a price functional implicitly based on a batch clearing system, or by a trading system generally based on a market-maker†. The basic intuition supported in these studies is that fundamentalists act as price stabilizers, by reducing the amplitude of price excursions, while trend chasers tend to amplify shocks. It has been shown that the interplay of these two groups of agents can induce short term non-normal price fluctuations, and can generate temporal patterns consistent with the statistical properties of the time series observed in real markets. These results suggest that the existence and permanence in the market of irrational or bounded rational traders is sufficient to explain the empirical evidence.

Recently, the role played by the set of rules governing the trading process has received growing attention. Agent-based simulations have embedded more realistic institutional settings and special attention has been devoted to analysing the impact of order-driven markets, either structured as continuous auction or as call auction systems (see Raberto et al. 2001, Chiarella and Iori 2002, Daniels et al. 2003, Li Calzi and Pellizzari 2003).

In a double auction order-driven market agents submit their request sequentially and transactions occur when two agents can match their request at a given price. Therefore, whenever an agent cannot find an opposite matching order, or the size of the matching order is lower than the required quantity, the agent will be rationed. Rationing has important consequences in terms of price dynamics. As underlined by Li Calzi and Pellizzari (2003), the continuous auction system increases the probability that incoming orders remain partly or totally unexecuted. Prices may not adjust to fully incorporate information/shocks as expressed by the trading requests, and they may tend to be stickier and to generate returns more concentrated around the mean. In addition, rationing may increase the probability of having sequences of traders on the same side of the market, and that in turn may imply a higher frequency of extreme price movements with respect to the average price change. Moreover, temporal patterns of dependence in financial time series may arise because rationed agents will keep on trying to satisfy their trading needs in subsequent trading sessions. The impact of rationing can be emphasized by introducing a more realistic setting where agents are subject to liquidity constraints. In such a case, agents' trading wishes can remain unsatisfied not only because of the lack of adequate opposite matching orders, but also because of the insufficiency of the available resources. Rationing may also occur in markets based on a batch clearing system, that is in markets where call auctions occur at regular time intervals during the trading day. Yet the phenomenon will tend to be less pervasive than in double auction market and, more importantly, it will not depend on the sequence of orders' arrival.

At this time, the analyses modelling explicitly the functioning of order-driven markets have provided some support to the conjecture that the fine structure of the trading mechanism plays an important role in determining the characteristics of financial time series.

Bak et al. (1997), Maslov (2000) and Li Calzi and Pellizzari (2003) model a double auction trading mechanism and show that the non-normality of the distribution of short term log-returns appears even when the market is populated by only one type of agent (typically pure noise traders or value traders). Raberto et al. (2001) model a call auction market and introduce an opinion propagation system that randomly creates clusters of agents trading on the same side of the market. They find both fat tails in the density of short term log-returns and volatility clustering in an artificial market with cash constrained noise traders, where agents submit buy or sell orders at limit prices dependent on past volatility. Their results depend on the mechanism inducing clusters of agents trading on the same side of the market and on the explicit connection imposed between current limit prices and past volatility.

The aim of our paper is to model an agent-based artificial market with a detailed representation of a continuous auction order-driven system, where multiple risky assets are exchanged $\$$. Agents, constrained by the available resources, trade to rebalance their portfolio. Agents are heterogeneous because they have to realize different target portfolio allocations, but they are homogeneous in terms of their trading strategies.

We build two different settings. In the first setting, agents are automatically rationed whenever they cannot realize their orders at current market conditions. In the second setting, we leave to the agents the possibility of choosing between posting market orders, at increasingly unfavourable conditions, and being rationed (and run the risk to remain rationed) but get more favourable trading terms in the case of a future trade. We make the assumption that the order submission strategy depends on the market conditions revealed by the order book structure. This setting represents a first step to make agents' behaviour dependent on market information. Agents will use the public information observable through the

†Additionally, agents' wealth is assumed unlimited (no liquidity constraints).

\$To our knowledge the only other paper where multiple risky assets are introduced is Cincotti et al. (2003). In their simulations the authors use the call auction market structure defined in Raberto et al. (2001). 
system (prices and volumes of the orders stored in the book) to infer order flow and price dynamics. In recent years, the diffusion of electronic limit order book systems has generated a growing literature analysing the links between the information observed through the system by traders, order placement behaviour and price dynamics (see Parlour 1998, Hall and Hautsch 2004, Handa et al. 2003, Ranaldo 2004). Based on the insights provided by this new research area, we expand our first setting, where both order flow and order type are exogenously determined, making order-type placement endogenous and dependent on the state of the book. In this way we introduce an explicit feedback mechanism between the intensity of rationing and price dynamics. We show that the state of the book provides an implicit coordination device inducing agents to supply liquidity (that is to submit limit orders) when the market needs it. Correspondingly, the rationing produced by the trading mechanism will be less intense, making both the nonnormality of the distribution of daily log-returns and the persistence of volatility less pronounced.

The extension to multiple risky asset is important for understanding the interactions between the budget constraint and the rationing effect induced by a double auction trading mechanism. When agents' purchases are limited by the available wealth and agents' portfolios are composed of multiple risky securities, at each moment in time, the agents' ability to satisfy their trading needs becomes related to market-wide conditions. Specifically, the realization of buy orders for the $i$ th asset will depend not only on the liquidity of the corresponding sell side of the order book, but also on the liquidity of the buy sides of the order books of the other risky assets. We show that, ceteris paribus, an increase in the number of assets traded in the market makes the distribution of daily log-returns more asymmetric around the mean. The negative skewness of the unconditional distribution of stock returns has been investigated empirically and assumed in many financial models (see Kraus and Litzemberger 1976, Cont 2001, Engle and Patton 2001, Hong and Stein 2003, Peirò 2004). In all the simulations we run we observe that the very largest price movements in the market are usually decrements rather than increments. Moreover, we find that the negative skewness of the distribution of stock returns becomes more pronounced when the parameters of the model are calibrated to generate more rationing. Our analysis suggests that the interaction of individual wealth constraints and the constraints imposed by the evolution of market liquidity plays an important role in making equity returns negatively skewed.

Finally, the multiple assets framework allow us to study the correlation structure of stock returns. We find significant positive correlations that increase in the settings where the rationing induced by the trading mechanism is stronger. We conjecture that the detected correlation structure is driven by positive wealth effects due to the traders' objective to maintain a well-balanced portfolio.

The paper is structured as follows. Section 2 introduces the market structure and the assumptions made in terms of agents' behaviour. Section 3 presents the calibration used for our simulations and discusses the results obtained. Section 4 concludes and describes future developments.

\section{The model}

\subsection{The market microstructure}

We use the following notation.

$M$ number of agents, with $i=1,2, \ldots, M$.

$N$ number of risky assets, with $j=1,2, \ldots, N$.

$T$ number of trading days, with $t=1,2, \ldots, T$.

$K$ number of time steps within trading day, with $k=1,2, \ldots, K$.

$C_{i}^{t}(k)$ amount of cash available at time step $k$, within the trading day $t$, and for trader $i$.

$P_{j}^{t}(k)$ price of risky asset $j$, at time step $k$, within the trading day $t$. Either the last transaction price or the last midquote if at the previous time step a midquote change occurred.

$B_{j}^{t}(k)$ best bid price for asset $j$, at time step $k$, within the trading day $t$.

$A_{j}^{t}(k)$ best ask price for asset $j$, at time step $k$, within the trading day $t$.

$W_{i}^{t}(k)$ total wealth for trader $i$, at time step $k$, within the trading day $t$.

$x_{i j}^{t}(k)$ number of shares of risky asset $j$, held by trader $i$, at time step $k$, within the trading day $t$.

$h_{i j}^{*}(t, t+\tau)$ target portfolio allocations for all assets including cash $(j=1,2, \ldots, N+1)$, for trader $i$, from period $t$ to $t+\tau$.

$q_{i j}^{t}(k)$ number of units of asset $j$ that agent $i$ is willing to trade at time step $k$ and trading day $t$.

We model an economy with $M$ agents where $N$ risky assets are exchanged, and the risk-free asset pays a zero yearly interest rate. The risk-free asset plays the role of cash available to the agents to satisfy their liquidity needs and its price is set equal to one.

We assume that borrowing and short selling are not allowed, therefore, $h_{i j}^{*}(t, t+\tau) \geq 0$ and $x_{i j}^{t}(k) \geq 0$. Agents allocate their total wealth entirely among the available assets, including cash, so we have that

$$
\sum_{j=1}^{N+1} h_{i j}^{*}(t, t+\tau)=1
$$

Agents get equal initial endowments in the $N+1$ existing securities. Given market prices $P_{j}^{t}(k) \geq 0$, the individual total wealth at each moment in time is

$$
W_{i}^{t}(k)=C_{i}^{t}(k)+\sum_{j=1}^{N} x_{i j}^{t}(k) P_{j}^{t}(k) \quad \text { for all } i=1,2, \ldots, M
$$

We randomly assign to each agent target portfolio allocations representing the fractions of total wealth that each agent desires to allocate in the existing securities. Given the restriction of non-negative portfolio allocations and 
given that portfolio allocations must exhaust all the available wealth, the set of target portfolios is given by

$$
D \equiv\left\{\mathbf{h}^{*}: \mathbf{h}^{\prime *} \mathbf{1}=1, \mathbf{h}^{*} \geq 0\right\} .
$$

To generate portfolios on the $N+1$ dimensional simplex $D$, we generate the target allocations as uniform variates Dirichlet $(1, \ldots, 1 ; 1)$ (see Gentle 1998). At regular time intervals we extract a subgroup of all agents and we assign to the agents of the group new target allocations. The duration of the assigned target allocations, $\tau$, is not defined ex-ante, but depends on the random sequence of extraction of each group.

Agents trade to rebalance their portfolio. At each moment in time they trade to adjust their portfolio according to their target allocations. We can interpret the change in agents' desired portfolio composition as the result of individual shocks (such as endowments shocks) hitting agents at random times, or as the result of the arrival of information of private interest (i.e. information about personal inventory costs, or about a private investment opportunity). As a consequence our agents can be regarded as uninformed or noise/liquidity traders.

At time step $k$, the number of units of the $j$ th asset that the $i$ th agent is willing to trade is given by

$$
q_{i j}^{t}(k)=\left\lfloor\frac{h_{i j}^{*}(t, t+\tau) W_{i}^{t}(k)-x_{i j}^{t}(k) P_{j}^{t}(k)}{P_{j}^{t}(k)^{\prime}}\right\rfloor,
$$

where $\lfloor\cdot\rfloor$ denotes the integer part and $P_{j}^{t}(k)^{\prime}$ is the effective price at which the transaction can be executed (respectively the best bid for sell orders and the best ask for buy orders). If $q_{i j}^{t}(k)>0$, the trader issues a buy order; if $q_{i j}^{t}(k)<0$, the trader issues a sell order.

Traders enter the market sequentially. At each time step $k$, we randomly extract (with replacement) one agent to enter the market. The selected agent will enter the market, and he will post his orders, if $P_{i}(E)$ is greater than a random number drawn from a uniform distribution over the $[0,1]$ interval. The probability $P_{i}(E)$ is an increasing function of the total imbalance between the target and the current portfolio,

$$
P_{i}(E)=f\left(\Delta_{i}\right)
$$

and

$$
\Delta_{i}=\sum_{j=1}^{N+1}\left|h_{i j}^{*}-\frac{x_{i j}^{t}(k) P_{j}^{t}(k)}{W_{i}^{t}(k)}\right| .
$$

The activation function $P_{i}(E)$ reflects the urgency of trading for the candidate agent. Agents are more impatient to trade, the more distant is their current wealth allocation from their target portfolio $\uparrow$. Correspondingly, the filtering device $P_{i}(E)$ makes the effective probability of entering the market dependent on the portfolio's imbalancet.

When a trader enters the market he faces an exchange book with orders to buy and to sell. Agents can trade immediately at the prevailing quote placing market orders, or they can submit limit orders that are stored in the exchange book and will be executed if matching orders arrive before the end of the trading day. Limit orders will be executed using first price priority and then time precedence. At each moment in time the exchange book shows all the orders that have been issued up to that time and that have not found a matching order. The book is divided in a buy side and a sell side. For each order, the order size, the limit price and the posting time are reported. The limit price is the maximum price that a buyer is willing to pay to purchase the listed quantity for a buy order, and the minimum price that a seller is willing to accept to sell the signed quantity for a sell order. At each moment in time the best/quoted bid, $B_{j}^{t}(k)$, is the highest limit buy price, that is the best price at which a seller can sell units of the asset. Conversely, the best/quoted ask, $A_{j}^{t}(k)$, is the lowest limit ask price, which is the best price at which a buyer can acquire units of the asset. Arriving orders are matched giving precedence to the registered orders with the best limit prices, and between orders with the same limit price, giving priority to the order with the lowest posting time.

Each day the opening price is the last spot price of the previous day. The spot price at each time step $k$ is either the last transaction price or the last midquote, if a change in the quotes occurred. Prices are discrete and, during each trading day, the minimum tick size change depends on the daily opening price. At the end of the trading day all orders are cancelled.

\subsection{Agents' behaviour}

We introduce the following notation to describe the agents' strategies:

$P_{j, b}^{t}(k)$ buying price of risky asset $j$, at time step $k$, within the trading day $t$;

$P_{j, s}^{t}(k) \quad$ selling price of risky asset $j$, at time step $k$, within the trading day $t$;

$M N_{i}^{t}(k)$ amount of money necessary to trade at time step $k$, within the trading day $t$, and for trader $i$;

$\dagger$ The relation between the urgency to trade and the size of the portfolio's imbalance can be explained as the result of the existence of fixed direct or indirect trading costs (such as a fixed fee that must be paid to access the market or the costs associated with the effort (and time)) needed to monitor and to adjust the portfolio's deviations.

$\$$ Given that in our model agents trade to reach a target portfolio, we introduce a device to transform a sequential random arrival process in an arrival process reflecting the agents' need for trading. We could have achieved the same result ordering, at each time step $k$, all the agents on the basis of $\Delta_{i}$, and then selecting the agents following that sequence. We have opted for the two step filtering procedure to minimize the computational burden. Clearly, with respect to a setting where the agents' arrival in the market is totally random, we are affecting the order-size distribution. Truncation from below generally increases the mean and decreases the dispersion of the probability distribution, therefore we can expect a similar effect on the mean and the dispersion of the order-type distribution. 
$M A_{i}^{t}(k)$ amount of money available for trading at time step $k$, within the trading day $t$, and for trader $i$;

$Q_{j}^{t}(k)$ number of units of asset $j$ available at the current quote, at time step $k$, and trading day $t$

$\epsilon^{t} \quad$ minimum tick size for trading day $t$;

$v$ upper bound on price deviation.

Agents trade to rebalance their portfolios subject to a budget constraint. We analyse two settings where agents adopt different order submission strategies: the simple trading and the simple trading with book analysis.

In the first setting the only order submission criterion is immediacy. The traders' objective is to satisfy their trading needs as soon as possible, thus they will submit market orders to buy (sell) at the current best ask (bid) for the quantities they need to trade. Limit orders are used only if for some $j$ the corresponding $q_{i j}^{t}(k)$ is greater than the quantity available at the current quote. In this case the agent places a market order for the quantity available, and for the residual quantity, given by $q_{i j}^{t}(k)-Q_{j}^{t}(k)$, he will submit a limit order to buy or to sell depending on the sign of $q_{i j}^{t}(k)$. The associated limit price will be such that the order will be first on the appropriate side of the book, so we have that

$$
\begin{aligned}
P_{j, b}^{t}(k) & =B_{j}^{t}(k)+\epsilon^{t}, \\
P_{j, s}^{t}(k) & =A_{j}^{t}(k)-\epsilon^{t} .
\end{aligned}
$$

When there are no orders on the relevant side of the book to match with, the agent will place directly a limit order for the whole quantity needed, $q_{i j}^{t}(k)$, at a price that will make him first on the book $\uparrow$.

Since borrowing and short-selling are not allowed, the agent's orders can be submitted only if the money needed to realize those trades is not greater than the money available. Let $I_{\{A\}}^{j}(k)$ be an indicator variable denoting for each risky asset $j$ if the agent wants to issue an order. The event $A$ can be a market order to buy (MB), a limit order to buy (LB), or a market order to sell (MS). To simplify the notation we drop the superscript indicating the trading day, then we can write:

$$
\begin{aligned}
M N_{i}(k)= & \sum_{j=1}^{N} I_{\{M B\}}^{j}(k) q_{j}^{\prime}(k) A_{j}(k) \\
& +\sum_{j=1}^{N} I_{\{L B\}}^{j}(k)\left[q_{i j}(k)-q_{j}^{\prime}(k)\right] P_{j, b}(k), \\
M A_{i}(k)= & C_{i}(k)-h_{i, N+1}^{*}(k) W_{i}(k)+\sum_{j=1}^{N} I_{\{M S\}}^{j}(k) q_{j}^{\prime}(k) B_{j}(k),
\end{aligned}
$$

where $q_{j}^{\prime}(k)=\min \left\{q_{i j}(k), Q_{j}(k)\right\}$ and $h_{i, N+1}^{*}(k)$ is the target cash allocation for the $i$ th agent.

Note that, $M N_{i}(k)$ is given by the amount of money necessary for market buy orders plus the amount of money required for limit buy orders, while, $M A_{i}(k)$ is equal to the amount of cash in excess of the target fraction of total wealth to keep as cash, plus the amount of money the agent can obtain from the sale of assets at market conditions. We do not include the money that could be realized through limit orders to sell because with respect to those orders the agent does not know if and when they will find a matching order. When agents submit their trades they must have in their hands all the money necessary to cover current expenses (the total value of market orders to buy) and future planned expenses (the total value of limit orders to buy submitted). If $M N_{i}(k) \leq M A_{i}(k)$, then all the orders that the agent wants to issue will be submitted. If $M N_{i}(k)>M A_{i}(k)$, then for each asset $j$ the number of units to trade are scaled down until $M N_{i}(k)=M A_{i}(k)$. The quantity adjustment keeps constant, with respect to the total, the percentage of money to allocate in each asset + .

The adjustments imposed by the budget constraint are performed giving priority to the submission of market orders to buy. If some money remains available after all market orders to buy have been processed, the procedure to check for the availability of money for submitting the desired limit orders to buy is started. Otherwise, the limit orders to buy are all cancelled.

In the simple trading with book analysis we allow agents to choose their order submission strategy. As in the previous setting, agents trade to rebalance their portfolio and they want to satisfy their trading needs as soon as possible. But, instead of imposing an automatic rationing, we allow agents to make their order submission strategy dependent on market conditions, as inferred from the public information revealed in the book. With respect to the previous setting, we are moving a step forward endogenizing the order-type placement.

In recent years, the relationship between the state of the limit order book of an order-driven market and the traders' order placement strategies has been studied both theoretically and empirically (see Parlour 1998, Hall and Hautsch 2004, Handa et al. 2003, Ranaldo 2004).

Parlour (1998) develops a dynamic model of an orderdriven market where traders can trade immediately by submitting market orders or choose a better price at the risk of non-execution by submitting limit orders. She shows that, even in the absence of asymmetric information and with a random arrival of traders types,

$\dagger$ The order fractioning rule that we impose is necessary to maintain a viable market with a regular flow of market and limit orders. In fact, an economy entirely populated by uninformed traders driven by liquidity needs (placing market orders until exhaustion of the orders stored in the book) will produce a degenerate market where one of the two sides of the book is systematically empty. In any case, the very simple rule of transforming 'excess' market orders in limit orders is not unusual in a real market. For instance, this is the procedure used in the electronic Milan Stock Exchange.

$\ddagger$ For each asset, the number of shares to purchase is computed as the integer part of the product of the original number of shares and the ratio between the money available and the money needed. The residual generated by the rounding procedure is automatically converted in cash. 
the optimal order strategy of individual agents depends on the state of the book (both sides of the book are determinant) and on the inferences about future order flow based on the current book. The important implication of the model is that the limit book provides a link between order flows in successive periods, and systematic patterns in transaction data will be observed.

Precisely, the thicker the bid (ask) side of the book and the thinner the ask (bid) side, the lower is the execution probability of a buy (sell) limit order. Therefore, traders are more likely to submit market orders when their own side of the book is thicker and the opposite side of the book is thinner.

Handa et al. (2003) model the price formation in a non-intermediated order-driven market with asymmetric information about the asset value. In their model the thickness of the buy and sell sides of the book is a proxy of the proportion of high and low valuation traders respectively. A higher proportion of high value (low value) investors raises the buy (sell) competition, making the execution probability of a limit buy (sell) order lower and a buy (sell) market order more attractive.

The empirical evidence is supporting the general hypothesis that trading behaviour is affected by the state of the book (see Hall and Hautsch 2003, Ranaldo 2004). In particular, a significant positive relation has been detected between market order submission and proxies measuring the differential thickness of the same side relative to the opposite side of the book.

Following these insights, we define a setting where agents use the information contained in the book to solve the trade-off between immediacy and price improvement. The first order submission criterion is immediacy: agents try to satisfy their trading needs at current market conditions submitting market orders (to buy or to sell) for the $q_{i j}(k)$. When the number of shares to trade is greater than the quantity available at the current quote, agents can choose between trading the residual quantity using market orders and submitting a limit order at a more favourable price.

Agents estimate the schedules relating cumulated buy/sell volumes to the corresponding bid/ask prices by fitting a linear relationship, using ordinary least squares $(O L S)$. If all the orders were collected in the book, the estimated schedules would represent the market demand and supply curves. The price at which the two schedules intersect would be the equilibrium price, $P_{j}^{\mathrm{E}}(k)$, that is the price at which the highest number of shares of the $j$ th asset could be traded. In our market the orders stored in the book are the orders that cannot be satisfied because the corresponding reservation prices are not compatible with the terms of trade expressed by the opposite side of the market (i.e. whenever $P_{j, s}(k) \leq P_{j, b}(k)$ a matching will occur). Arriving market orders sequentially remove orders from the opposite side of the book. Thus, we can interpret the estimated schedules, starting from the current quotes, as the truncated unsatisfied demand and supply curves given current market conditions. The price $P_{j}^{\mathrm{E}}(k)$ is the limit price starting from which, for any given volume, the terms of trade of buyers and sellers do not allow any exchange $\left(A_{j}(k)>B_{j}(k)\right)$. The proxy used by our agents, to combine information provided by the book with inferences about the order flow, is the differential distance between the current quotes and the reference price $P_{j}^{\mathrm{E}}(k)$. Let us denote by $z_{j}^{\mathrm{B}}(k)$ and $z_{j}^{\mathrm{A}}(k)$, respectively, the distance between the reference price and the current bid, and the distance between the current ask and the reference price,

$$
\begin{aligned}
& z_{j}^{\mathrm{B}}(k)=P_{j}^{\mathrm{E}}(k)-B_{j}(k), \\
& z_{j}^{A}(k)=A_{j}(k)-P_{j}^{\mathrm{E}}(k) .
\end{aligned}
$$

The order submission strategy will work as follows.

(i) $z_{j}^{\mathrm{B}}(k)>z_{j}^{\mathrm{A}}(k)$, agents infer that in the market there is more selling than buying. A buyer will choose to trade the residual quantity submitting a limit order to buy at an improved price,

$$
P_{j, b}(k)=B_{j}(k)-\Delta_{j}
$$

where

$$
\Delta_{j}=\left\{\begin{array}{cl}
z_{j}^{\mathrm{B}}(k)-z_{j}^{\mathrm{A}}(k) & \text { if } z_{j}^{\mathrm{B}}(k)-z_{j}^{\mathrm{A}}(k)<v, \\
v & \text { if } z_{j}^{\mathrm{B}}(k)-z_{j}^{\mathrm{A}}(k) \geq v .
\end{array}\right.
$$

A seller will choose to trade the residual quantity submitting market orders until there are limit orders to buy to match with (she will walk down the bid book). She will issue a limit order to sell, at a price such that she will be first on the book, only if a residual quantity remains after all limit orders to buy existing in the book have been matched.

(ii) $z_{j}^{\mathrm{B}}(k)<z_{j}^{\mathrm{A}}(k)$, agents infer that in the market there is more buying than selling. A seller will choose to trade the residual quantity submitting a limit order to sell at an improved price,

$$
P_{j, s}(k)=A_{j}(k)+\Delta_{j},
$$

where,

$$
\Delta_{j}=\left\{\begin{array}{cc}
\left|z_{j}^{\mathrm{B}}(k)-z_{j}^{\mathrm{A}}(k)\right| & \text { if }\left|z_{j}^{\mathrm{B}}(k)-z_{j}^{\mathrm{A}}(k)\right|<v, \\
v & \text { if }\left|z_{j}^{\mathrm{B}}(k)-z_{j}^{\mathrm{A}}(k)\right| \geq v .
\end{array}\right.
$$

A buyer will keep on submitting market orders to match with existing sell limit orders (he will walk up the ask book). He will issue a limit order to buy, at a price such that he will be first on the bid book, only if a residual quantity remains after all sell limit orders in the book have been matched.

(iii) $z_{j}^{\mathrm{B}}(k)=z_{j}^{\mathrm{A}}(k)$, agents cannot make any inference about the prevalent direction of the order flow. In this case they will trade the residual quantity posting limit orders at prices such that they will be first on the book.

When the buy and sell schedules cannot be estimated, because there are less than two limit orders on at least one side of the book, agents make their inferences about market conditions comparing cumulated ask and bid volumes. The budget constraint operates as in the previous setting, giving priority to market orders when an adjustment is necessary. 
Table 1. Simulation parameters. The symbol $\sim$ denotes an independent draw from a probability distribution.

\begin{tabular}{lll}
\hline Number of agents & $M=10000$ & \\
Number of risky assets & $N=1,3,6$ & \\
Number of trading days & $T=5000$ & $i=1,2, \ldots, M$ \\
Number of daily time steps & $K=360$ & \\
Initial cash endowments & $C_{i}^{1}(1)=\frac{M C}{M}$ & $i=1,2, \ldots, M$ \\
Initial endowments of risky assets & $x_{i j}^{1}(1)=50$ & $j=1,2, \ldots, N$ \\
Initial prices & $P_{j}^{1}(1)=€ 100$ & $j=1,2, \ldots, N$ \\
Number of groups & $G=10,20,50$ & $i=1,2, \ldots, M$ \\
Interval for updating allocation vectors & $I=10,20,30,40$ days \\
Target allocation vectors & $\mathbf{h}_{i}^{*} \sim$ Dirichlet $(1, \ldots, 1 ; 1)$ & if $0 \leq \Delta_{i} \leq 0.3$ \\
Tick size & $\epsilon^{t}=1 \%$ opening price \\
Entering probability & & if $0.3<\Delta_{i} \leq 0.6$ \\
& $f\left(\Delta_{i}\right)= \begin{cases}0.5 \Delta_{i} \\
1.83\left(\Delta_{i}-0.3\right)+0.15 \\
0.75\left(\Delta_{i}-0.6\right)+0.7 & \text { if } 0.6<\Delta_{i} \leq 1 \\
1 & \text { if } \Delta_{i}>1\end{cases}$ \\
Upper bound on price changes & $v=5 \epsilon^{t}$ &
\end{tabular}

\section{Simulation results}

We run our simulations ${ }^{\dagger}$ with a population of $M=10000$ potentially active traders, $T=5000$ trading days and $N=1,3,6$ risky assets. Each trading day is divided in $K=360$ time steps corresponding, approximately, to a trading day of six hours, assuming a time step $k$ equal to one minute. Each agent gets an initial endowment in cash equal to the market capitalization divided for the total number of agents in the market $(M C / M)$. The individual starting endowments in each of the $N$ stocks of our economy are set equal to 50 shares. Initial prices are set equal to $€ 100$. Agents are divided in $G$ equally sized groups with $G=10,20,50$. Every $I$ days, with $I=10,20,30,40$, one group is randomly extracted and all the agents in the selected group get new target allocations $h_{i j}^{*}(t, t+\tau)$. Target allocation vectors, $\mathbf{h}_{i}^{*}$, are sampled from a Dirichlet $(1, \ldots, 1 ; 1)$. For the $i$ th trader, the probability of entering the market, $P_{i}(E)=f\left(\Delta_{i}\right)$, is determined as followst,

$$
f:[0,2] \rightarrow[0,1]
$$

with

$$
f\left(\Delta_{i}\right)= \begin{cases}0.5 \Delta_{i} & \text { if } 0 \leq \Delta_{i} \leq 0.3 \\ 1.83\left(\Delta_{i}-0.3\right)+0.15 & \text { if } 0.3<\Delta_{i} \leq 0.6 \\ 0.75\left(\Delta_{i}-0.6\right)+0.7 & \text { if } 0.6<\Delta_{i} \leq 1 \\ 1 & \text { if } \Delta_{i}>1\end{cases}
$$

We use two different ways to assign new target allocations: the uniform and the non-uniform updating. In the uniform case, the new target allocations are equal for all the agents in the group. We extract a new allocation vector for the first agent of the selected group and then we assign the same allocation vector to all the other agents in the group. In the non-uniform case, we extract a different allocation vector for all the agents in the group. The minimum tick size is $1 \%$ of the opening daily price, while we set the upper bound on price change equal to five ticks. See table 1 for a summary of the parameters used in the simulations.

\subsection{Analysis of the short term log-returns distribution}

We choose a standard parameters' calibration where $G=20, I=40$ and $N=3$. In figure 1 we plot the typical daily time series of prices, volume and volatility generated in the simple trading and in the simple trading with book analysis under the two scenarios of uniform and nonuniform updating§. We systematically discard the first 1000 observations to avoid spurious effects due to the initialization of the simulations. The daily price series reports for each day the last transaction price registered during the trading day. Daily volatility is defined as the squared first difference in the daily log-prices.

At first inspection, it seems that the main difference in the behaviour of prices over time is due to the technique used to update target allocations. In fact, in both settings, when we move from non-uniform to uniform updating we get (i) price series with a higher range of variation and (ii) price series showing some trends over time and less pronounced continuous up and down movements. Furthermore, in the case of uniform updating, the

$\dagger$ The artificial market is implemented using C ++ language, with MatClass libraries (Birchenhall, 1993).

$\$$ The total portfolio's imbalance reaches the maximum when the agent's wealth is entirely allocated on one asset and it must be reallocated on another asset (in this case, the sum in absolute value of the changes occurred will be two). The probability function we specify yields a stretched $S$-shaped function. We choose this functional form because of its flexibility. The function is calibrated to obtain an entering probability equal to one when the total imbalance is at least $50 \%$.

§We show the series corresponding to a single asset generated in a three risky assets economy under the specified combination of parameters. 

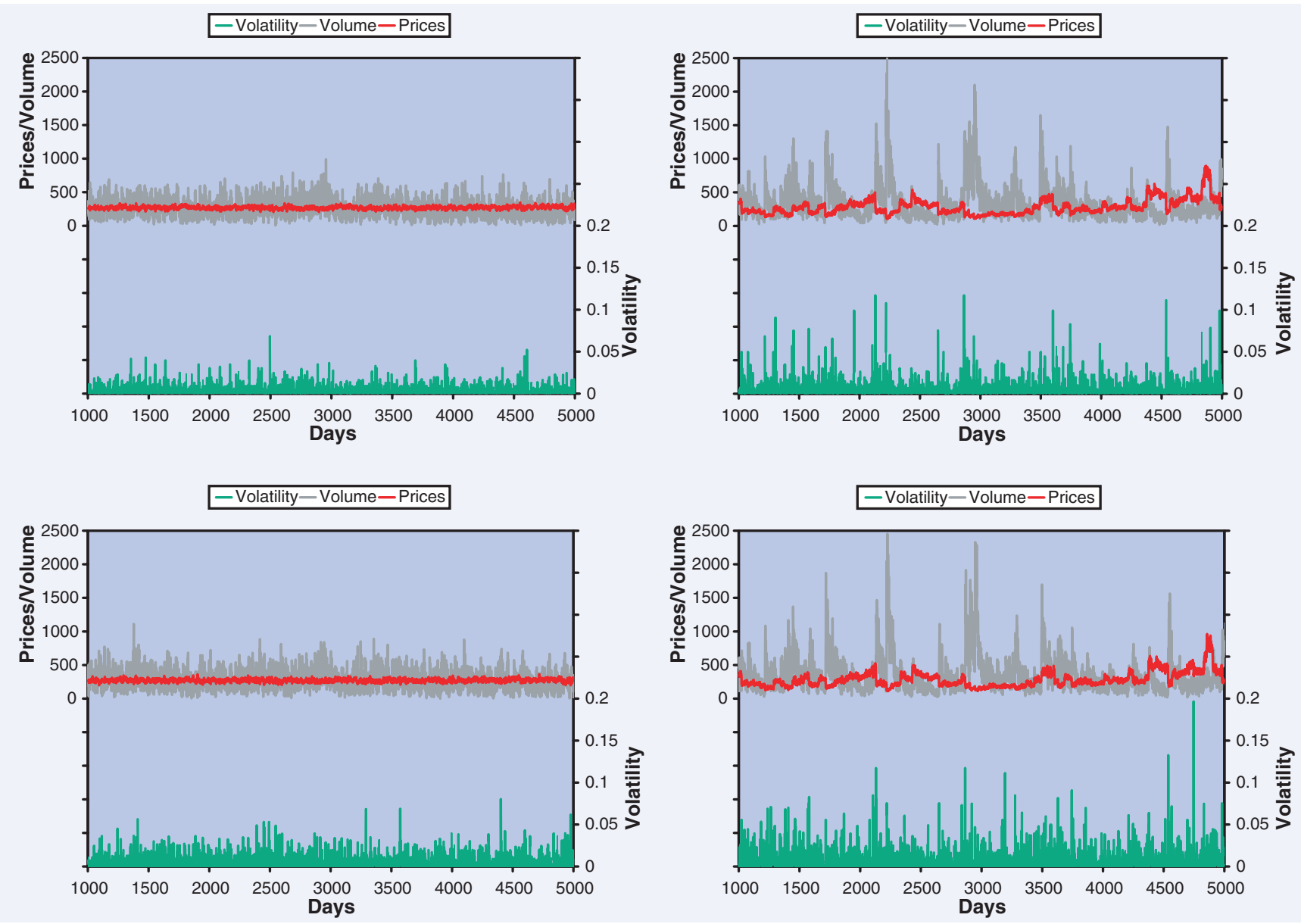

Figure 1. Daily time series of prices, volume and volatility for the different settings. From the upper-left to the right-bottom panel (clockwise), we have: simple trading with non-uniform updating (STNU), simple trading with uniform updating (STU), book analysis with non-uniform updating (BANU) and book analysis with uniform updating (BAU).

temporal pattern of price changes appears much more concentrated on small values with the occurrence of single high spikes.

When we look at the shape of the distribution of daily stock returns, we observe that all our series show evidence of excess kurtosis and negative skewness. In all the simulations the Shapiro-Wilk test rejects normality at a significance level less than $1 \% \dagger$.

To highlight the behaviour of the tails of the distribution, in figure 2 we show the cumulative distribution of the standardized log-returns. We use the absolute value of the standardized log-returns in order to merge the positive and negative tail. In all cases there is evidence of fat tails with some important differences. The nonnormality is more pronounced in the cases of uniform updating and, independently of the method used to change target allocations, in the simple trading setting.

We check for the robustness of these results, with respect to the parameters specifying the updating time interval and the number of groups. To this end, we run for each parameters' combination $(G=10,20,50$ and
$I=10,20,30,40)$ a set of simulations corresponding to ten different seed numbers. For sufficiently long updating time intervals, we reject the normality hypothesis, and the daily standardized log-returns show fat tails, excess kurtosis and negative skewness. To gather some insights about the factors driving the observed phenomena, we analyse the change in the behaviour of the series related to different combinations of the parameters. In figures 3 and 4 we report the values of the total tail's slope, corresponding to the simple and the book-analysis setting in the two updating regimes.

The comparative analysis highlights some interesting patterns.

(i) There is a clear trend towards more pronounced fat tails associated with longer time-intervals between one updating and the other.

(ii) In the uniform updating regime, the deviations from normality become stronger the lower the number of groups.

$\dagger$ Over twenty simulations for each market setting (maintaining $G=20$ and $I=40$ ), the kurtosis statistic is consistently higher in the uniform updating regime and in the simple trading setting. Equivalently, the skewness coefficient becomes more negative in the uniform case and in the simple trading setting. 

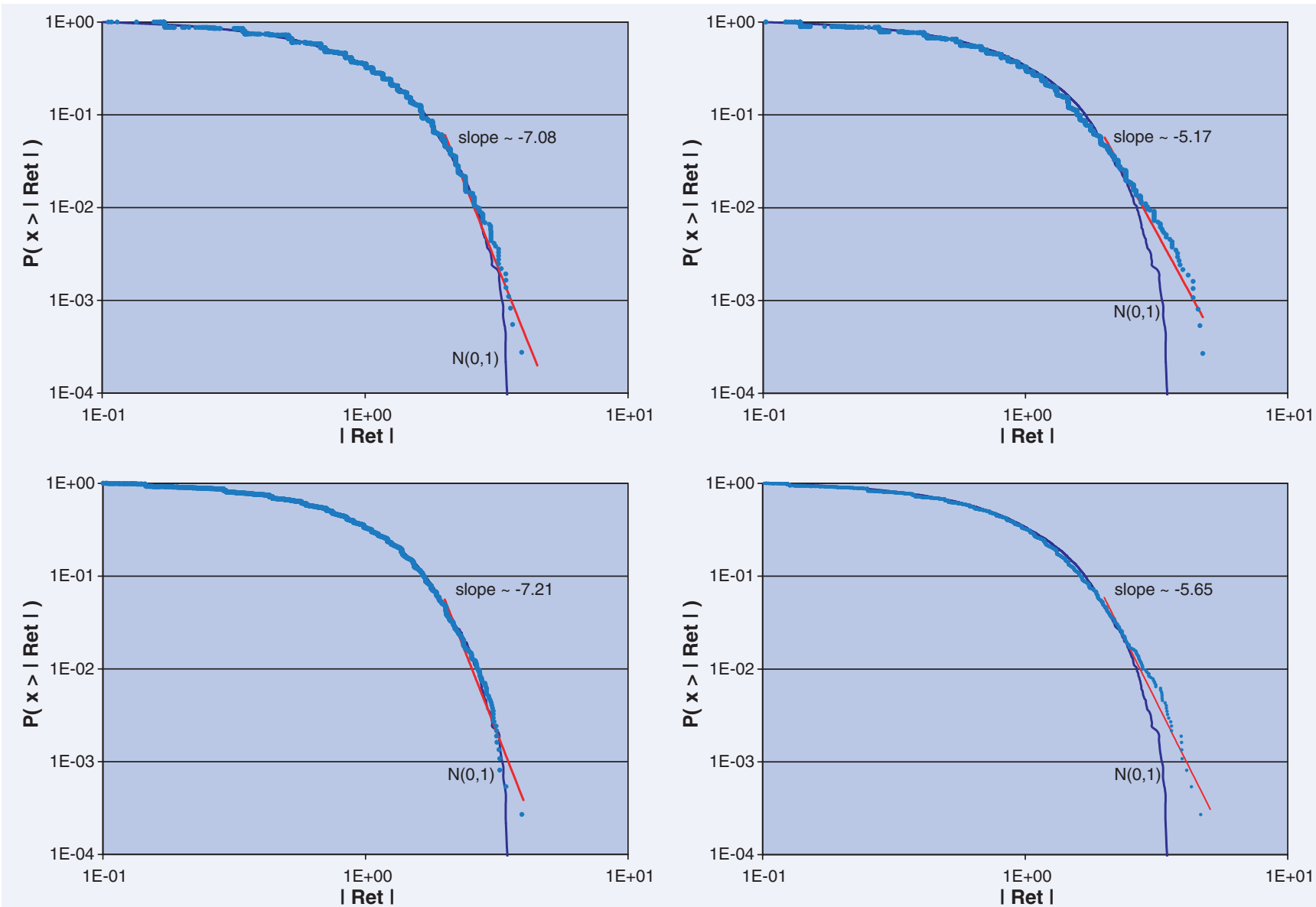

Figure 2. Cumulative distribution of the standardized log-returns for the STNU, STU, BANU and BAU settings (clockwise). The fat tails of the log-returns distribution are more pronounced in the two right panels corresponding to the case of uniform updating and, independently of the method used to update the target allocations, in the simple trading setting.

(iii) For each combination of parameters, the deviations from normality are more evident in the uniform than in the non-uniform setting.

(iv) The above patterns are general, but in the bookanalysis framework, we observe an attenuation of the deviations from normality.

The identified patterns make clear the role played by the heterogeneity of the population in affecting the shape of log-returns distribution.

In the uniform updating setting, at each updating time, we assign the same target allocations to all the agents in the selected group. Clearly in this case, the lower the number of groups and the longer the time interval between one updating and the other, the lower is the degree of heterogeneity of the agents' population. When the population is more homogeneous, the probability of having sequences of agents on the same side of the market increases. Since in a double auction market trading requests can be satisfied only if they find an opposite matching order, the trading mechanism will tend to produce an accumulation of unsatisfied orders on one side of the book. That in turn, will tend to generate more frequent extreme price changes $\uparrow$. In the non-uniform setting, the impact of changing the number of groups on the degree of heterogeneity of the population is less evident: while typically the lower is the updating frequency the stronger are the deviations from normality. In the book

$\dagger$ The price dynamics induced by this trading mechanism is strictly related to the sequences of order flow (in terms of direction, buy or sell, and size). A well-balanced population, where $50 \%$ of the agents are buyers and the remaining $50 \%$ are sellers for a compatible aggregate quantity, will support a steady state price in a call auction market but not in a double auction market. What matters here is the sequential arrival of traders. When the probability of having sequences of traders on the same side of the market increases, there will be an accumulation of limit orders that will freeze the market. The arrival of a sequence of traders willing to trade in the opposite direction will eventually produce a big price jump.

fActually, under this regime, for sufficiently long updating time interval, the population becomes less heterogeneous when the number of groups is high. In this case, when a group is extracted a small number of agents will receive different target allocations. Given the low extraction probability of each group, if the updating frequency is low enough, a high proportion of the population will converge towards the target portfolio. Thus, a large number of agents will tend to trade in the same direction when prices change. 
oSTU_10ロSTU_20 $\Delta$ STU_50

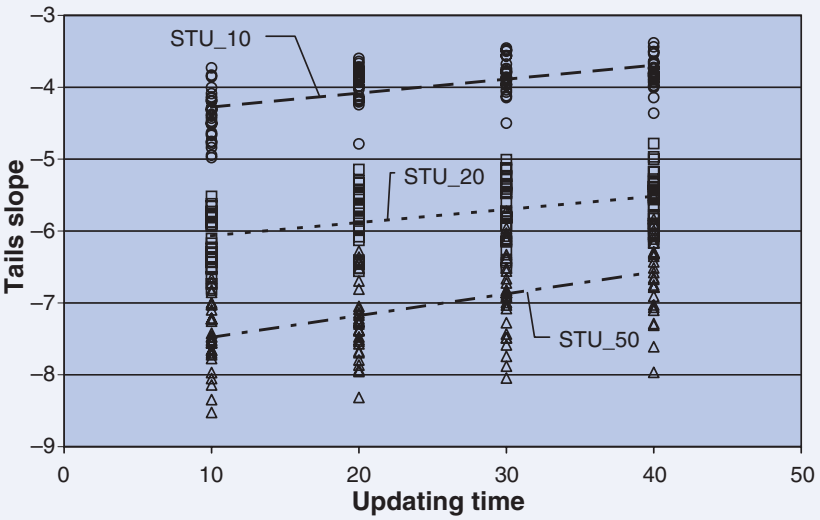

oBAU_10BAU_20 $\Delta$ BAU_50

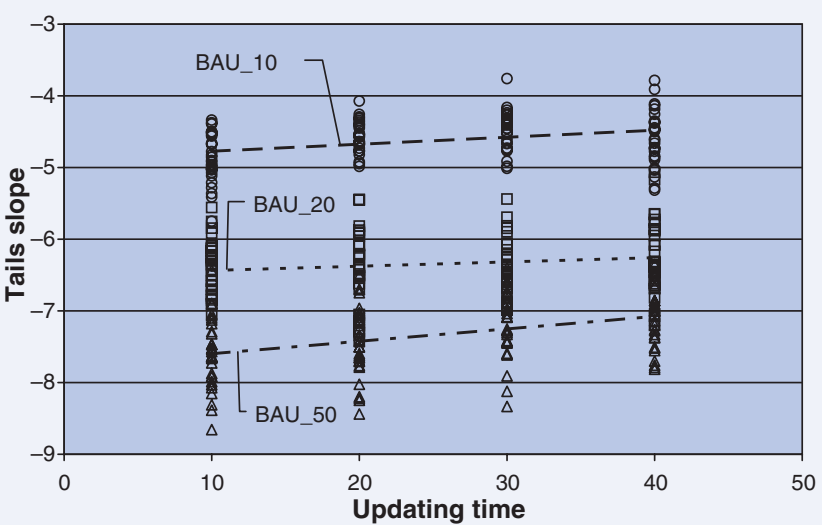

OSTU_10ロSTU_20 $\Delta$ STNU_10×STNU_20

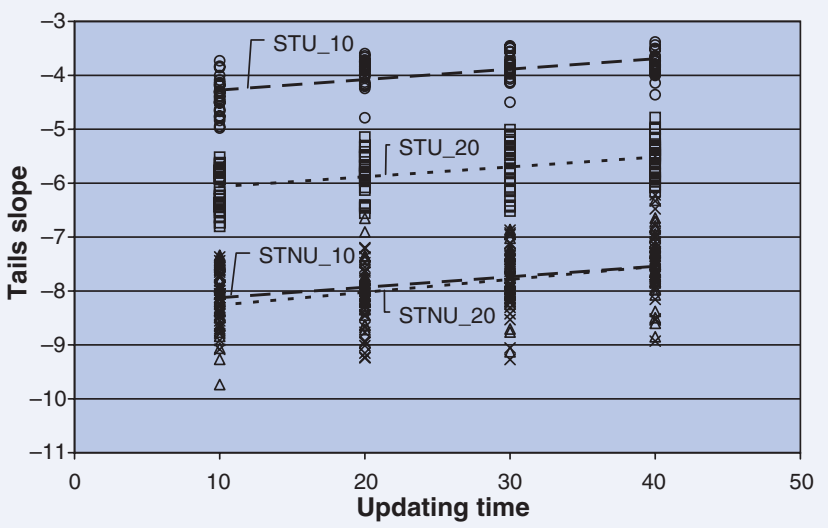

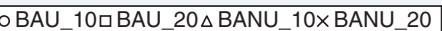

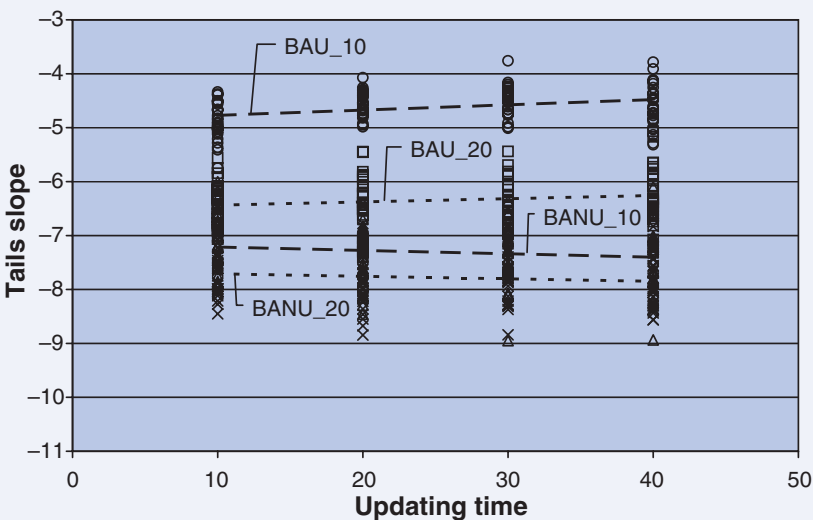

Figure 3. The left panels report the coefficient measuring the slope of the merged tails of the cumulative distribution of standardized log-returns for the STU and the BAU settings. The slope coefficients are computed for the series generated in an economy with three risky assets. For every combination of parameters $(G=10,20,50$ and $I=10,20,30,40)$, we report the values for the three assets in ten simulations (thirty points). We can see a clear increasing trend both with respect to longer updating time intervals and with respect to a reduction in the number of groups. The two right panels compare the uniform and the non-uniform regimes, and show that the fat tails become more pronounced in the uniform updating case.

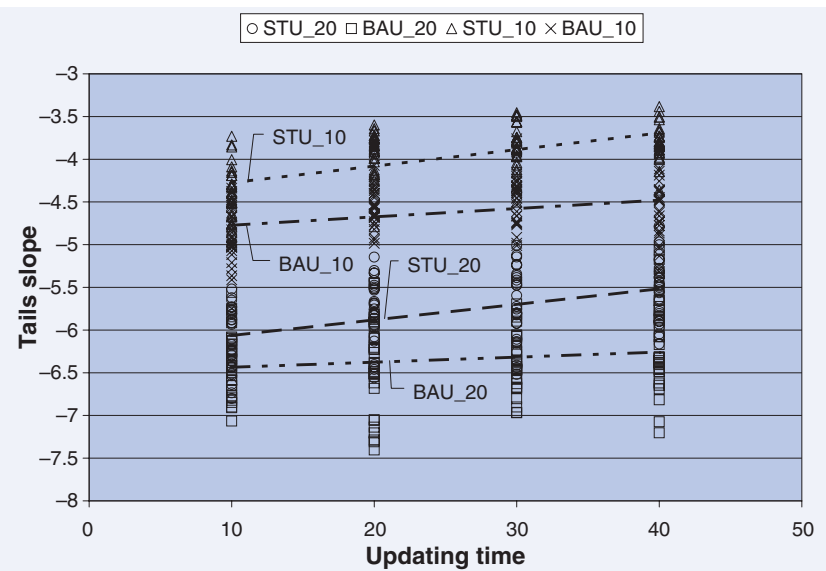

Figure 4. Tail slope coefficients computed under the uniform regime for ten series generated in an economy with three risky assets for $G=10,20$ and $I=10,20,30,40$. The comparison shows that the fat tails of the log-returns distribution increase when moving from the book analysis to the simple trading framework.

analysis setting agents can choose between fully satisfy their trading needs at market prices and post a limit order (i.e. incur rationing). The choice depends on the inferences they make about market imbalance. The
Table 2. Average daily spread computed, for each asset in every setting, over twenty simulations with parameters $G=20$ and $I=40$.

\begin{tabular}{lrrrr}
\hline Model & Asset $_{1}$ & Asset $_{2}$ & Asset $_{3}$ & Average \\
STNU & 5.563 & 5.564 & 5.556 & 5.561 \\
STU & 5.875 & 5.864 & 5.987 & 5.909 \\
BANU & 17.847 & 17.840 & 17.922 & 17.870 \\
BAU & 19.114 & 19.100 & 19.650 & 19.288 \\
\hline
\end{tabular}

agents' order-type submission strategy makes the accumulation of limit orders on one side of the market less pronounced. Therefore, rationing will occur less frequently and, with respect to the simple trading setting, we will observe more frequent, and wider, price changes around the average and less frequent extreme jumps. In table 2 we report the average daily spreads of twenty simulations with $G=20, I=40$ and $N=3$. We can clearly see that the average daily spread increases substantially in the book analysis setting.

In figure 5 we report separately the left and right tail of the distribution of standardized log-returns generated under uniform updating in the simple and in the bookanalysis setting. We notice that the typical log-returns 

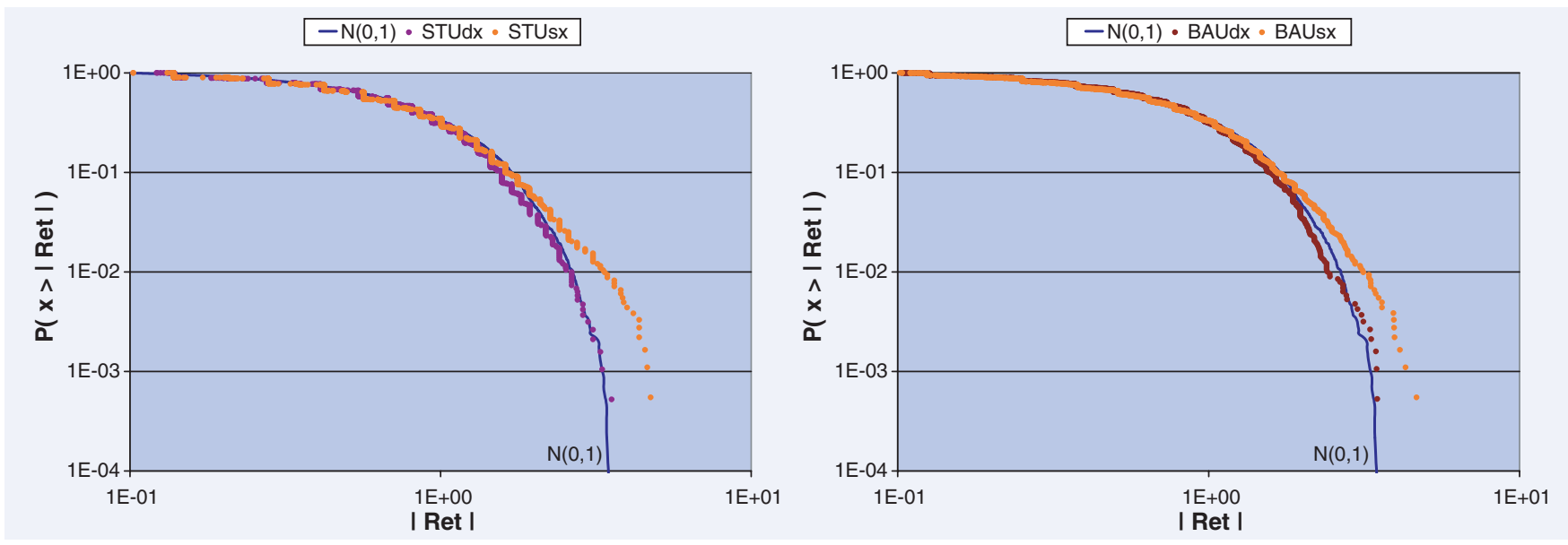

Figure 5. Cumulative distribution of standardized log-returns for the STU and the BAU settings showing left and right tail separately.

series generated with our simulator presents a tendency towards a more pronounced low tail.

The asymmetry of the unconditional distribution of stock return has been widely debated both empirically and theoretically. The empirical evidence documents negative skewness and asymmetric volatility of stockmarket returns (see Gallant et al. 1992, Chen et al. 2001, Engle and Patton 2001), though the pervasiveness of the phenomenon is still an open question (see Peirò 2004). On the theoretical side, most of the proposed explanations assumed a representative-investor framework $\uparrow$. Recently Hong and Stein (2003) have provided an explanation based on investor heterogeneity. In their model the negative skewness of market returns is determined by the interaction of differences of opinion about the fundamental value of the assets and market frictions due to short sales constraints. Interestingly, their model implies a relation between trading volume and asymmetric volatility.

As stressed by Peirò:

'[...] departures of the coefficient of skewness from zero must be understood as rejections of normality, rather than rejections of asymmetry [...] in order to test for symmetry distribution-free procedures should be used.'

(Peirò 2004, p. 38)
We implement two different non-parametric tests. First, we examine the whole density function of returns computing the excess returns with respect to the mean and then comparing the distribution of negative excess returns with the distribution of positive excess returns. We divide absolute excess returns in seven classes and we perform a likelihood ratio test to verify if the two groups of data are significantly different:. We perform the test on all the simulated series and we always reject the null at a significance level not greater than $1 \%$.

To look closely at the tails of the distribution we follow the procedure suggested by Peirò (2004). We select the twenty most extreme excess movements, and we test the null hypothesis that the probabilities of the most extreme returns being negative or positive are not different\$. We run the test on the series generated in twenty simulation runs of the simple trading setting with uniform updating, three assets, $G=20$ and $I=40$. In all cases but two (58 series) we get a $p$ value lower than 0.05 . We run the same test changing the number of risky assets of the economy. In figure 6 we compare the $p$ values of the case of one single risky asset, with those relative to the case of three and six risky assets respectively.

Interestingly, we observe that the $p$ values decrease as the number of risky assets increases. Thus, the evidence suggests that, ceteris paribus, the asymmetry of the tails

†We are referring to the leverage effects theory (Black 1976, Christie 1982), to the volatility feedback mechanism (Pindyck 1984, Campbell and Hentschel 1992) and to the bubble models (Blanchard and Watson 1982).

tThe null hypothesis that the two groups are sampled from the same population is tested using the following statistic,

$$
T=2 \sum_{i} \sum_{j} n_{i j} \log \left(\frac{n_{i j}}{m_{i j}}\right),
$$

where $n_{i j}$ is the observed number of cases categorized in the $i$ th row of the $j$ th column, while $m_{i j}$ is the number of cases expected in the $i$ th row of the jth column when $H_{0}$ is true. The values of the statistic are distributed asymptotically as a $\chi^{2}$ with $\mathrm{d} f=(r-1)(c-1)$, where $r$ and $c$ are respectively the number of rows and the number of columns of the contingency table (in our test $r=2$ and $c=7$ ). $\S$ The $p$ value of the test is computed as follows,

$$
p=2\left(1-\Phi\left(\frac{\max \left(n^{-}, n^{+}\right)+0.5-n p}{\sqrt{n p(1-p)}}\right)\right),
$$

where $n^{-}$and $n^{+}$are the number of negative and positive excess returns, while $\Phi$ is the cumulative standard normal distribution function. 

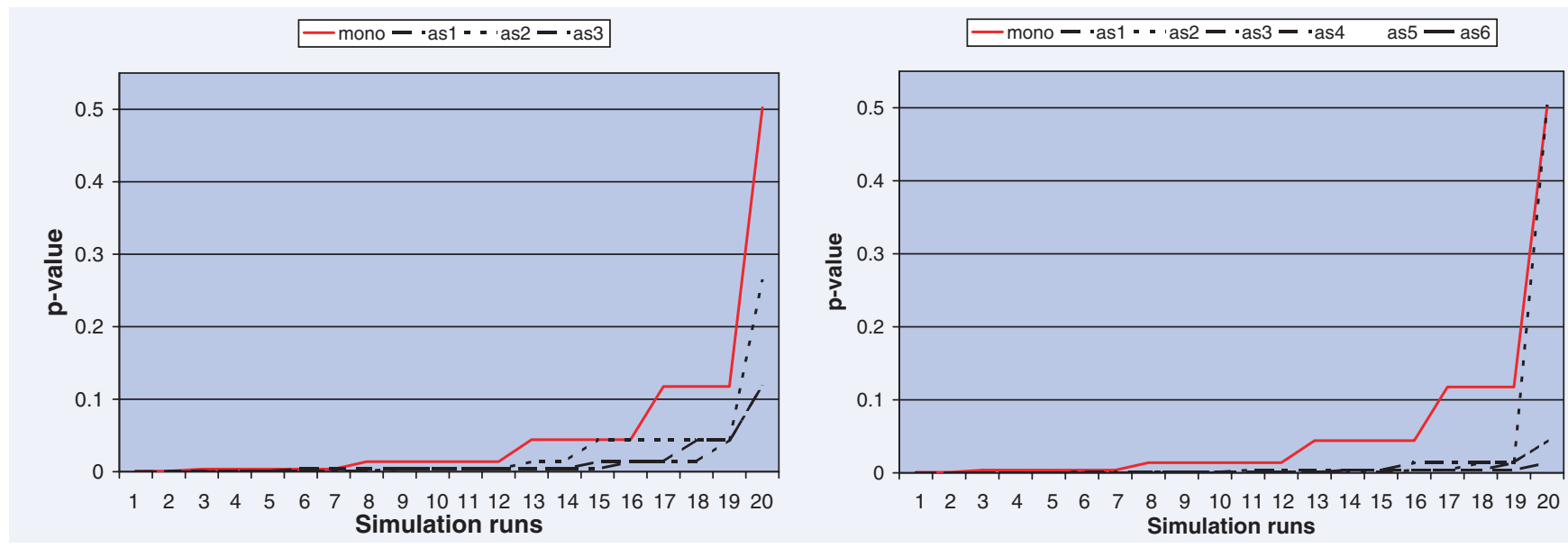

Figure 6. Sorted $p$ values of the tails asymmetry test for twenty simulation runs of the STU setting with $G=20$ and $I=40$. The continuous line represents the case of a market with one single risky asset. The dotted lines correspond to the $p$ values generated in the case of a three risky asset economy (left panel) and in the case of an economy with six risky assets (right panel).

of the stock returns distribution becomes more pronounced as the number of assets included in the agents' portfolios increases.

We conjecture that the negative skewness (specially in the tails of the distribution) is due to the interplay between agents financial constraints and the frictions generated by the trading mechanism. As a matter of fact, in our model, jumps in prices and returns are determined by the arrival of sequences of traders on the same side of the book. Big positive/negative jumps occur with the arrival of sequences of buyers/sellers that match with a series of different sell/buy limit orders. Clearly, two conditions must be verified to get big jumps: there must be several limit orders listed in the book and the corresponding quantities must be relatively small with respect to the arriving contrarian market orders. Otherwise, even if a sequence of agents willing to trade on the same side of the market arrives, traders will tend to match with the first few limit orders and no big deviation in price will be observed. Since the budget constraint can scale down or even cancel buy orders while it does not affect sell orders, for a given distribution of target allocations across agents, sell limit orders will tend to be more numerous and for bigger quantities than buy limit orders. Moreover, the order size of sell limit orders relative to the order size of opposite market orders will be on average bigger than the relative order size of buy limit orders. Therefore, downside price movements will tend to happen more often than upside price changes. In addition, since the down and up movements, respectively on the bid and ask side of the book, depend on the order size of arriving opposite market orders, the negative skewness will increase with trading volume.

The statistics reported in table 3 confirm our expectations. The average order size of limit orders to sell is higher than the average order size of limit order to buy. Moreover, the ratio of the average order size of limit orders to sell with respect to contrarian market orders is higher than the corresponding ratio of limit orders to buy with respect to market orders to sell. The above
Table 3. For $G=20$ and $I=40$ and for each market setting, the table reports the ratios between the mean per-order volume of limit to contrarian market orders ( $L O S / M O B$ and $L O B / M O S)$, and the ratios representing the mean per-order volume of buy and sell limit orders (VLOS/NLOS and $V L O B / N L O B)$. For each asset we compute the mean over 4000 days. The values reported are averages over twenty simulation runs. For the STU setting we report the values corresponding to an economy with one single risky asset, and to a multi-securities economy with three and six risky assets respectively.

\begin{tabular}{l|ccccc}
\hline Model & Asset & LOS & LOB & VLOS & VLOB \\
& & /MOB & /MOS & /NLOS & /NLOB \\
\multirow{4}{*}{ STNU } & 1 & 2.61 & 2.56 & 32.77 & 27.00 \\
& 2 & 2.61 & 2.58 & 32.77 & 27.01 \\
& 3 & 2.61 & 2.57 & 32.69 & 27.04 \\
\cline { 2 - 6 } & 1 & 2.00 & 2.01 & 20.67 & 19.00 \\
\cline { 2 - 6 } & 1 & 2.33 & 2.23 & 24.12 & 19.23 \\
& 2 & 2.36 & 2.21 & 24.59 & 19.33 \\
& 3 & 2.34 & 2.25 & 23.58 & 18.85 \\
\cline { 2 - 6 } STU & 1 & 2.53 & 2.37 & 25.43 & 18.97 \\
& 2 & 2.56 & 2.37 & 26.35 & 19.37 \\
& 3 & 2.51 & 2.33 & 25.33 & 18.65 \\
& 4 & 2.47 & 2.32 & 24.25 & 18.56 \\
& 5 & 2.52 & 2.30 & 24.88 & 18.06 \\
& 6 & 2.54 & 2.35 & 26.18 & 19.20 \\
\hline
\end{tabular}

differences increase when moving from the single risky asset case to the case where portfolios are composed of six risky assets and in the uniform case.

To examine the behaviour of volatility, in figure 7 , we plot the price changes with respect to classes in increasing order of standardized log-volume. We observe that, as we move to volume classes corresponding to bigger deviations from the average volume, the volatility of price changes increases and the relative frequency of big negative price changes goes up.

The pervasiveness of the non-normality of the daily log-returns time series, together with the qualitative and quantitative differences detected in the proposed 


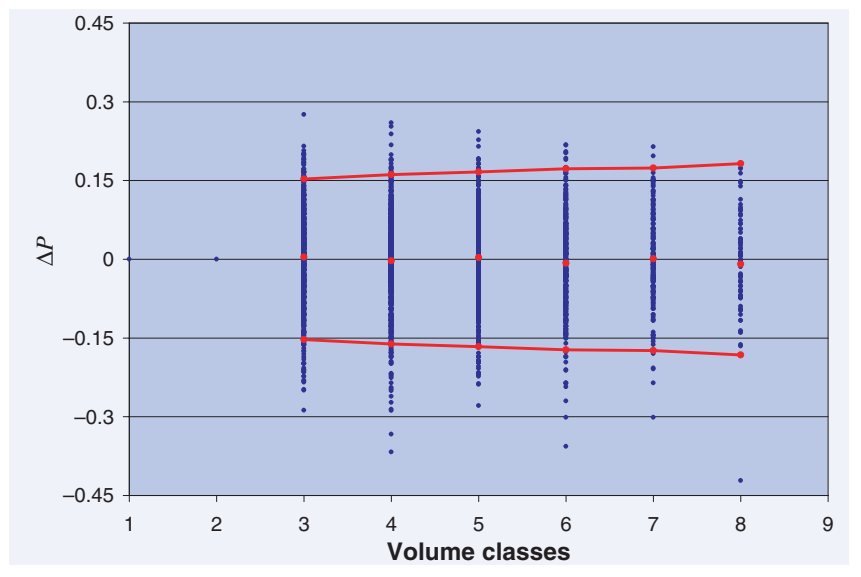

Figure 7. Log-price changes, for the BAU setting, relative to volume classes in increasing order of standardized log-volume: (1) $v_{i} \leq-3$, (2) $-3<v_{i} \leq-1.5$, (3) $-1.5<v_{i} \leq-0.5$, (4) $-0.5<v_{i} \leq 0, \quad$ (5) $0<v_{i} \leq 0.5, \quad$ (6) $0.5<v_{i} \leq 1.5, \quad$ (7) $1.5<v_{i} \leq 3$ and (8) $v_{i} \geq 3$.0. For each volume class, we report the mean price change and the interval corresponding to $\pm 2 \sigma$.

Table 4. Average Spearman correlation coefficient between daily returns. The average is taken, for each asset in every setting, over twenty simulations with parameters $G=20$ and $I=40$.

\begin{tabular}{lccc}
\hline Model & $\rho_{12}$ & $\rho_{13}$ & $\rho_{23}$ \\
STNU & 0.11 & 0.12 & 0.13 \\
STU & 0.18 & 0.20 & 0.19 \\
BANU & 0.08 & 0.07 & 0.10 \\
BAU & 0.11 & 0.12 & 0.11 \\
\hline
\end{tabular}

alternative settings, strongly supports the idea that the institutional environment defined by a continuous auction order-driven market affects significantly the distribution of short term log-returns. The price dynamics depends on the intensity of rationing induced by the trading mechanism†.

Our analyses show that there is a clear relation between the shape of the distribution of daily log-returns and the intensity of rationing generated by the sequential matching trading mechanism. In particular, the negative asymmetry of the log-returns distribution is strictly related to the interplay between individual wealth constraints and the liquidity constraints generated by the market mechanism.

Finally, we examine the stock returns correlation structure. In table 4 we report the average Spearman correlations over twenty simulations with parameters $G=20, N=3$ and $I=40$.

We notice two interesting facts. First, the average correlations are significantly positive at least at the $1 \%$ level. Second, the correlations increase in the settings where the induced rationing is stronger, that is in the simple trading framework and under the uniformupdating regime.

In our model with uninformed traders and random exogenous order flow, the only way for an association structure to arise is through the wealth effect due to the traders' objective of maintaining well-balanced portfolios:. When in the market there is more rationing, a higher proportion of traders will maintain over time $\Delta_{j}$ 's significantly different from zero and of the same sign. The wealth effect will cumulate across agents and produce a stronger aggregate impact on prices.

\subsection{The temporal pattern of the simulated financial time series}

We analyse here the temporal properties of the simulated series. For all our series the Augmented Dickey-Fuller $(A D F)$ test rejects the null hypothesis of a unit root in the log-returns series. To check for $I I D$, or at least uncorrelated, log-price changes we perform the variance ratio test $(V R)$. For all the series the $V R$ statistic computed at different lags is significantly lower than one, rejecting the random walk hypothesis§. Correspondingly, the LjungBox Q-test consistently rejects the hypothesis of no serial correlation in the time series of log-returns.

Figures 8 and 9 show the sample autocorrelations at different lags of the volatility and the volume time series. All the series present significant serial correlations at different lags which become more pronounced when moving from the non-uniform to the uniform case. Moreover, the volume series show a clear tendency towards an accentuation of the temporal dependence in the simple trading setting.

The log-returns series, figure 10, presents negative short-term serial correlations that tend to become stronger in the book analysis setting.

When the randomly assigned target allocations are the same for all the agents in the selected group, large demand/supply imbalances become more probable. Rationed agents will keep issuing similar orders until they can find a match, thus creating memory effects in volume that are amplified by an increase in the intensity of the induced rationing. The temporal behaviour of the log-returns and volatility series can be explained by the interaction of two different forces. On the one hand, rationing implies that prices do not fully adjust to reflect market imbalances. Accordingly, prices will continue to adjust through time generating persistence in volatility. Over time the propagation effect will be more persistent

\footnotetext{
$\dagger$ Our conjecture is further supported by the result we obtain when we run our simulations without the entering probability. As we mentioned above, the second step filter we impose increases the mean and reduces the dispersion of the order size probability distribution. Accordingly, the sequential matching of orders will produce less rationing. As expected, we observe an attenuation of the non-normality of the stock returns distribution when we introduce the entering probability.

$\ddagger$ For every agent, for every asset $j, \Delta_{j}$ increases when the price of another asset goes up, $\partial \Delta_{j} / \partial P_{i} \geq 0$ for $i \neq j$.

$\S$ We use both the standard variance ratio test and the heteroskedasticity-consistent variance ratio test, and we always get results consistent with rejection of the null hypothesis of random walk price increments.
} 

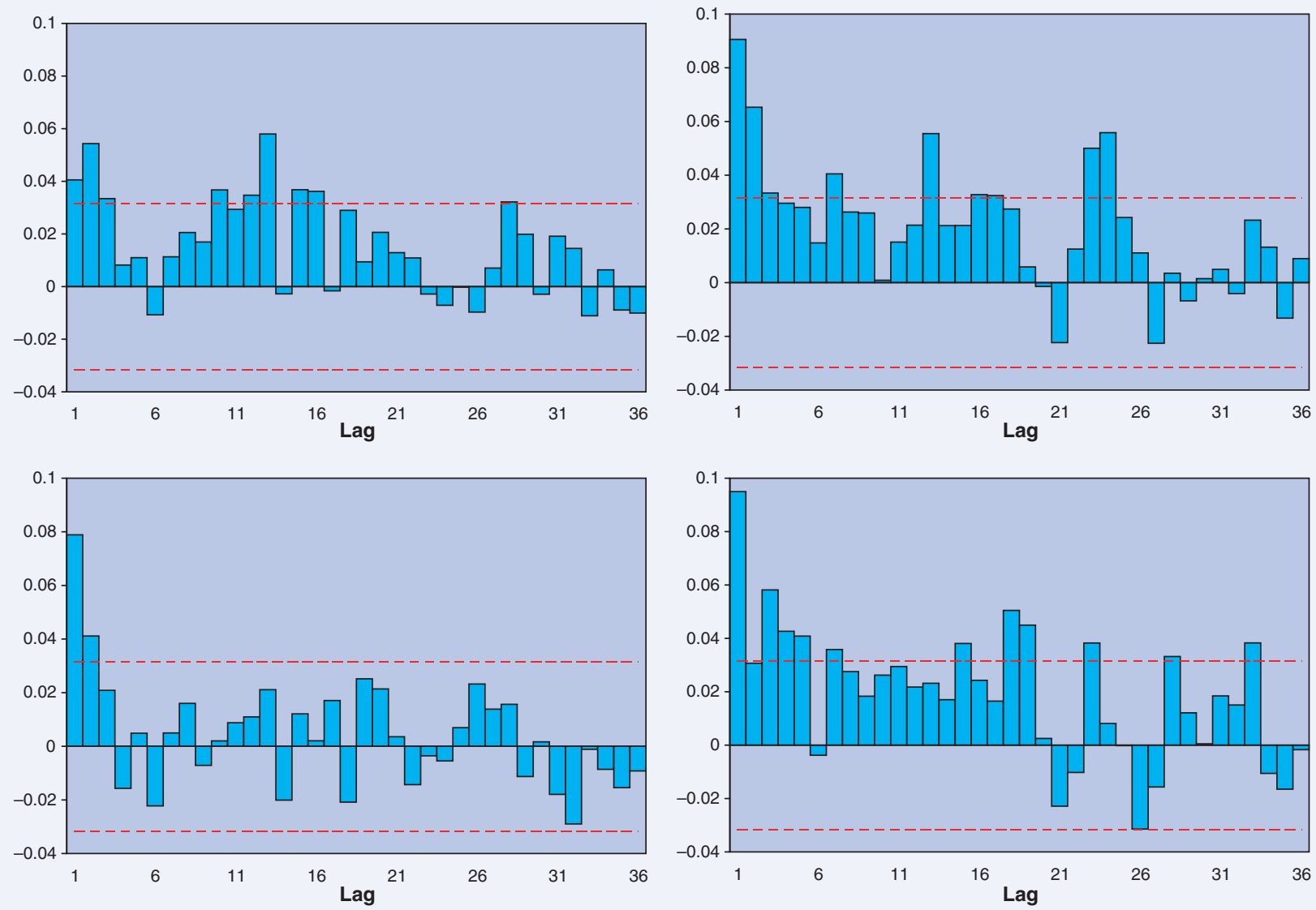

Figure 8. Autocorrelation function of volatility for STNU, STU, BANU and BAU (clockwise). The volatility series are more persistent in the uniform updating cases.
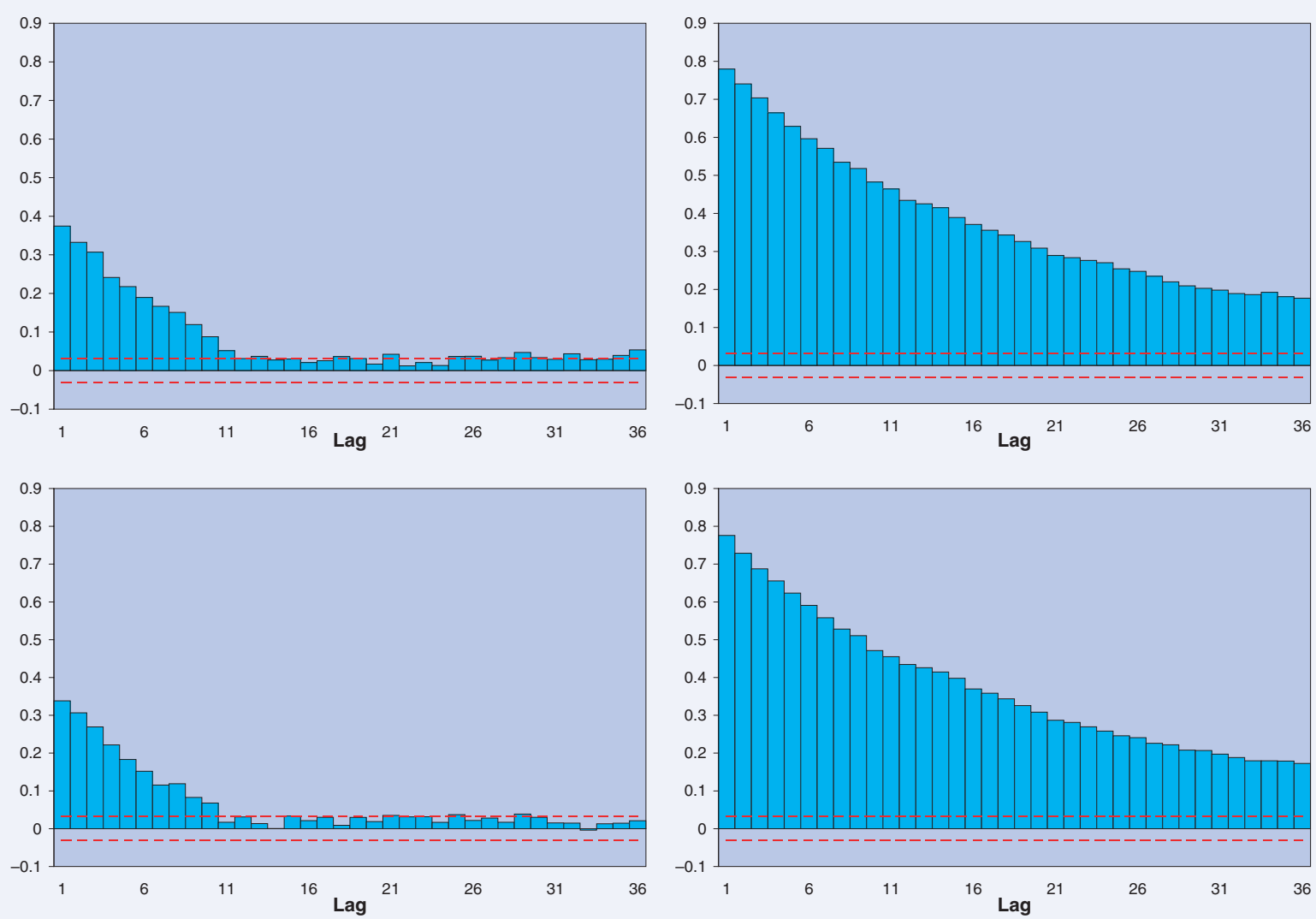

Figure 9. Autocorrelation function of volume for STNU, STU, BANU and BAU (clockwise). The serial correlation of volume becomes higher in the uniform updating case and is more pronounced in the simple trading setting. 

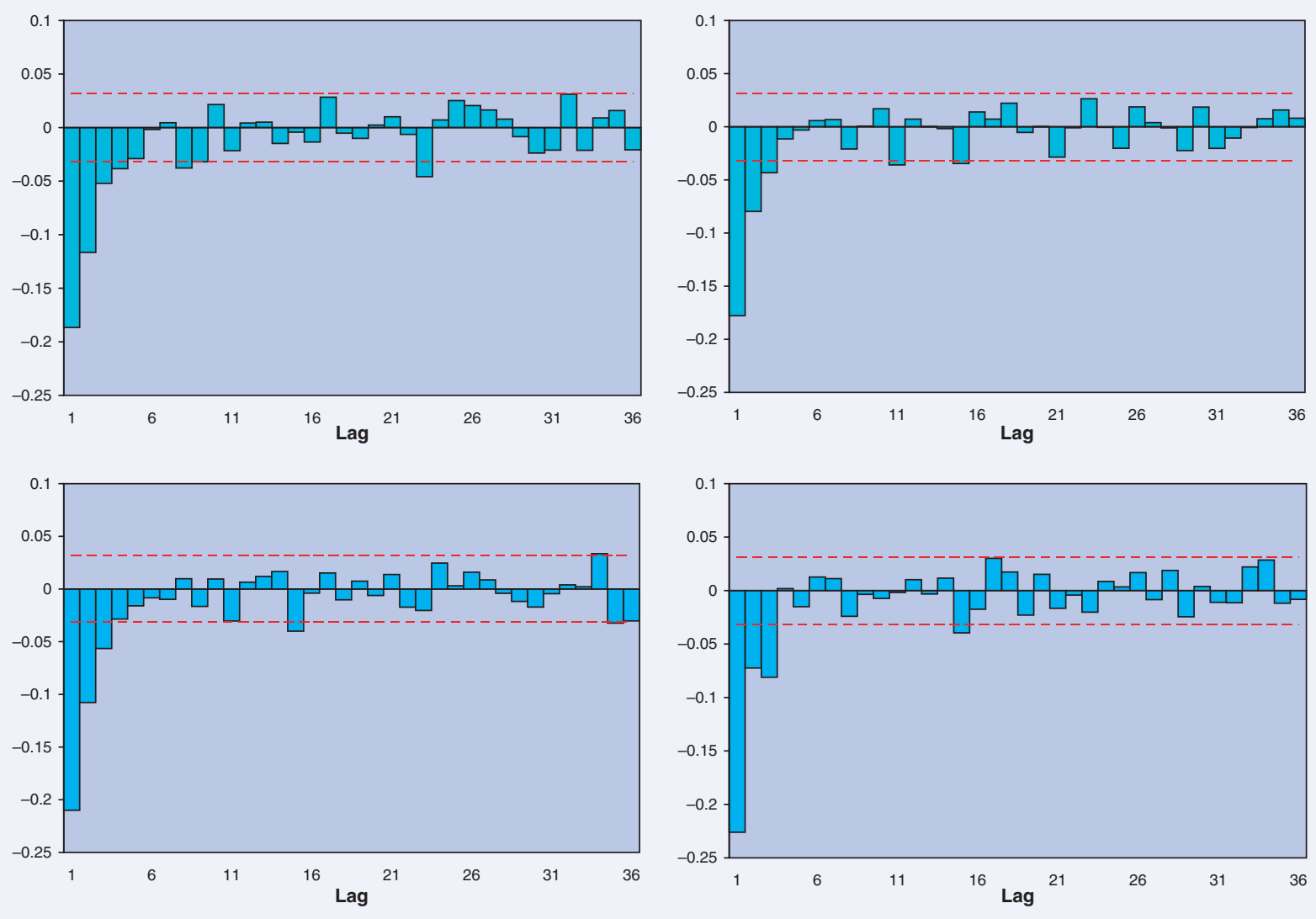

Figure 10. Autocorrelation function of log-returns for STNU, STU, BANU and BAU (clockwise). We note that the negative serial correlation in log-returns becomes more pronounced in the book analysis setting (bottom panels).

in those settings where rationing is more intense. On the other hand, since agents trade to adjust their portfolio allocations according to target allocations, when, for a given asset, the price increases, for all agents the difference between the amount that should be invested in that asset and the amount currently invested decreasest. Therefore, big price movements will induce contrarian trades that, in turn, will generate short-term negative serial correlation in log-returns and positive short-term correlation in volatility. The short-term serial correlation of log-returns and volatility is more pronounced in the book analysis setting where the average bid-ask spread is higher and the magnitude of price fluctuations is greater.

In figure 11 we plot the sample cross-correlations between volume and the absolute value of log-price changes. Clearly, the persistence of the cross-correlation over time is closely related to the intensity of the rationing induced by the trading mechanism.

\section{Conclusions}

We have modelled an artificial financial market with multiple risky assets where agents trade to reach target portfolio allocations exogenously assigned, subject to a budget constraint. Agents are homogeneous in terms of trading strategy, nevertheless the market is maintained viable by assigning to the agents different target allocations over time. The trading mechanism replicates the functioning of a continuous auction order-driven market.

We show that the institutional setting of a double auction market is sufficient to generate a non-normal distribution of price changes and temporal patterns that resemble those observed in real markets.

We can summarize our results as follows.

(a) The daily log-returns series we generate exhibit fat tails, excess kurtosis and negative skewness. The deviations from normality become more evident in those setting where the rationing induced by the trading mechanism is stronger (the simple trading setting and the uniform updating case). Therefore, we provide significant evidence supporting the idea that the institutional structure of the market plays an important role in determining the distribution of short-term price changes. The generated distributions of log-returns become closer to those observed in real markets, when we reduce agents' heterogeneity, creating groups of agents with similar trading needs.

Our model suggests that it is not necessary to introduce heterogeneity in agents' trading strategies to generate the non-normality of the log-returns

$\dagger \partial \Delta_{j} / \partial P_{j} \leq 0$ for $j=1,2, \ldots, N$. 

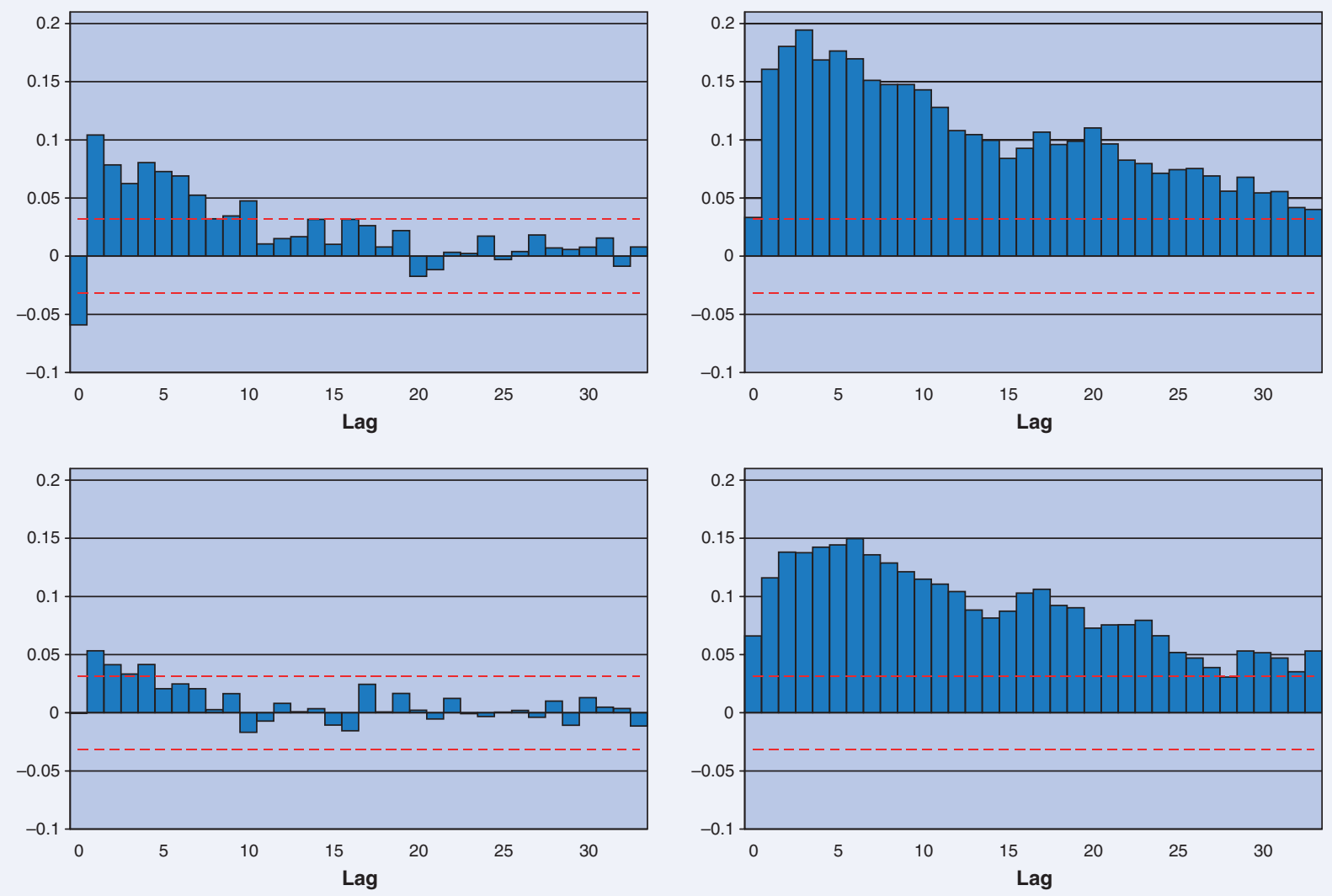

Figure 11. Cross-correlations of volume and absolute value of price changes for STNU, STU, BANU and BAU (clockwise). The cross-correlations are more persistent in the uniform updating case and in the simple trading setting.

distribution. The sequential matching system of a double auction market can by itself produce anomalous price dynamics when the random order-flow is such to increase the probability of having sequences of traders on the same side of the market.

(b) Our multi-assets framework makes it possible to derive some interesting insights about the role of the budget constraint. We find evidence of negative skewness and a more pronounced low tail of the daily distribution of log-returns, and we show that the phenomenon is related to the interplay between agents' financial constraints and the frictions generated by the trading mechanism. In addition, even in our framework where order-flow is exogenous and independent of market relevant information, we detect positive returns correlations associated with the intensity of rationing induced by the trading system.

(c) Our model generates financial time series whose temporal pattern resembles the actual behaviour of real financial time series: serial correlation in volatility, in volume, and positive cross-correlation between volume and the absolute value of price changes. The detected temporal patterns become more pronounced in the market settings where the intensity of rationing induced by the trading mechanism increases.

In closing, we underline that the current framework represents a basic set-up where we examined how prices respond to order-flow in a double auction market. In our multi-assets model order-flows are exogenously determined and do not depend on information about fundamentals or market-wide conditions. Of course, in reality order-flow rates depend on information and assumptions about agents' utility. To develop a sensible multiple securities market model, the connections between agents' choices and the process of information production and diffusion must be addressed. We have chosen to follow a two-step procedure to attack such a complex issue.

First, we have examined how a multi-assets works for given order-flow rates. Interestingly, we find that a multiassets market presents peculiar characteristics even in this simplified setting. We highlight that the interaction of the trading mechanism with wealth constraints creates some feedbacks affecting significantly the stock returns distributions of single risky assets. The introduction of the book-analysis setting allowed us to move in the direction of modelling the information flow and to qualify our results. Allowing agents to choose to be liquidity demanders or liquidity suppliers on the basis of the public screen information, we have performed the first step to switch from a model where agents' behaviour is random and exogenously determined to a model where agents' choices are dependent on information and based on economic criteria.

The final step requires to make order-flow dependent on information. We are working to introduce endogenously determined target allocations. In this new setting, investors will consider the past to be informative about 
the future, and they will determine their target allocations on the basis of their estimates of expected prices conditional on current information. In particular, we are defining a framework where agents learn about the joint probability distribution of assets returns, and consequently determine optimal portfolio allocations. Such a framework will open the possibility to analyse the dynamics of aggregate stock-market returns and to investigate how market-wide price movements, and related contagion effects, can arise.

\section{Acknowledgments}

AC acknowledges MIUR for partial support under research grant PRIN 2004 'Modelli per la dinamica dei prezzi di titoli finanziari: aspetti istituzionali ed ipotesi comportamentali in un'ottica agent-based'.

\section{References}

Bak, P., Paczuski, M. and Shubik, M., Price variation in a stock market with many agents. Physica A, 1997, 246, 430-453.

Birchenhall, C., A draft guide to MatCLass. A matrix class for $\mathrm{C}++$. Technical Report, 1993 (University of Manchester).

Black, F., Studies of stock market volatility changes, in Proceedings of the American Statistical Association, Business and Economics Section, 1976, pp. 177-181.

Blanchard, O. and Watson, M., Bubbles, rational expectations, and financial markets. In Crises in Economic and Financial Structure, edited by P. Wachtel, pp. 295-315, 1982, (Lexington Books: Lexington MA).

Brock, W., Hommes, C. and Wagener, F., Evolutionary dynamics in financial markets with many trader types. Working Paper 7, 2001 (Wisconsin Madison-Social Systems, University Of Wisconsin Madison).

Campbell, J. and Hentschel, L., No news in good news: an asymmetric model of changing volatility in stock returns. J. Financ. Econ., 1992, 31, 282-318.

Campbell, J., Lo, A. and MacKinlay, A., The Econometrics of Financial Markets, 1997 (Princeton University Press: Princeton NJ).

Chen, J., Hong, H. and Stein, J., Forecasting crashes: trading volume, past returns, and conditional skewness in stock prices. J. Financ. Econ., 2001, 61, 345-381.

Chen, S., Lux, T. and Marchesi, M., Testing for non-linearity structure in an artificial market. J. Econ. Behav. Organ., 2001, 46, 327-342.

Chiarella, C. and He, T., Asset price and wealth dynamics under heterogeneous expetations. Quant. Finance, 2001, 1, 509-526.

Chiarella, C. and Iori, G., A simulation analysis of the microstructure of double auction markets. Quant. Finance, 2002, 2, 346-353.
Christie, A., The stochastic behavior of common stock variances: value, leverage, and interest rate effects. J. Financ. Econ., 1982, 10, 407-432.

Cincotti, S., Focardi, S., Marchesi, M. and Raberto, M., Who wins? Study of long-run trader survival in an artificial stock market. Physica A, 2003, 324, 227-233.

Cont, R., Empirical properties of asset returns: stylized facts and statistical issues. Quant. Finance, 2001, 1, 223-236.

Daniels, G.M., Farmer, D.J., Gillemot, L., Iori, G. and Smith, E., Quantitative model of price diffusion and market friction based on trading as a mechanistic random process. Phys. Rev. Lett., 2003, 90, 108102

Engle, R. and Patton, J., What good is a volatility model? Quant. Finance, 2001, 1, 237-245.

Farmer, D. and Joshi, S., The price dynamics of common trading strategies. J. Econ. Behav. Organ., 2002, 49, 149-171.

Gallant, R., Rossi, P. and Tauchen, G., Stock prices and volume. Rev. Financ. Stud., 1992, 5, 199-242.

Gentle, J., Random Number Generation and Monte Carlo Methods, 1998 (Springer: Berlin).

Hall, A. and Hautsch, N., A continuous-time measurement of the buy-sell pressure in a limit-order book market. Discussion Paper, 2004 (University of Copenhagen).

Handa, P., Schwartz, R. and Tiwari, A., Quote setting and price formation in an order driven market. J. Financ. Markets, 2003, 6, 461-489.

Hong, H. and Stein, J., Differences of opinion, short-sales constraints, and market crashes. Rev. Financ. Stud., 2003, 16, 487-525.

Karpoff, J., The relation between price changes and trading volume: a survey. J. Financ. Quant. Anal., 1987, 22, 109-126.

Kraus, A. and Litzenberger, R., Skewness preference and the valuation of risky assets. J. Finance, 1976, 31, 1085-1100.

LeBaron, B., Building Financial markets with artificial agents: desired goals and present techniques. In Computational Markets, edited by G. Karakoulas, 1999 (MIT Press: Cambridge, MA).

LeBaron, B., Empirical regularities from interacting long and short memory investors in an agent-based stock market. IEEE Trans. Evol. Comput., 2001, 5, 442-455.

Li Calzi, M. and Pellizzari, P., Fundamentalists clashing over the book: a study of order-driven stock markets. Quant. Finance, 2003, 3, 470-480.

Maslov, S., A simple model of a limit order-driven market. Physica A, 2000, 278, 571-578.

Pagan, A., The econometrics of financial markets. J. Empirical Finance, 1996, 3, 15-102.

Parlour, C., Price dynamics in limit order markets. Rev. Financ. Stud., 1998, 11, 789-816.

Peirò, A., Asymmetries and tails in stock index returns: are their distributions really asymmetric? Quant. Finance, 2004, 4, 37-44.

Pindyck, R., Risk, inflation, and the stock market. Am. Econ. Rev., 1984, 74, 334-351.

Raberto, M., Cincotti, S., Focardi, S. and Marchesi, M., Agent-based simulation of a financial market. Physica A, 2001, 299, 319-327.

Ranaldo, A., Order aggressiveness in limit order book markets. J. Financ. Markets, 2004, 7, 53-74.

Routledge, B., Adaptive learning in financial markets. Rev. Financ. Stud., 1999, 12, 1165-1202. 